\title{
¿QUÉ ES "EDUCACIÓN DE CALIDAD” PARA DIRECTORES Y DOCENTES?
}

\author{
Pablo Torche ${ }^{2}$ \\ Javiera Martínez $z^{3}$ \\ Javiera Madrid ${ }^{4}$ \\ Javier Araya ${ }^{5}$
}

RESUMEN

La presente investigación ofrece un modelo preliminar para comprender la forma en que directores y profesores conciben el concepto de educación de calidad, el cual fue levantado a partir de las opiniones cualitativas de directores y profesores de 12 establecimientos del país. El modelo propuesto distingue tres niveles de profundidad para organizar los componentes de una educación de calidad. Los dos niveles más profundos incluyen componentes relacionados con el compromiso personal y afectivo con los estudiantes así como la formación valórica y de habilidades para la vida, en tanto que solo el tercero incorpora los resultados de aprendizaje y las variables de gestión escolar. A la luz de este modelo es posible comprender de mejor manera la forma en que los actores educativos se relacionan con las estrategias de evaluación de la calidad (particularmente el Simce), y otras políticas educativas relacionadas.

Palabras clave: educación de calidad, calidad de la educación, evaluación de calidad, formación valórica, gestión escolar, Simce.

\section{HOW IS "QUALITY EDUCATION" DEFINED BY PRINCIPALS AND TEACHERS?}

\section{ABSTRACT}

This research offers a preliminary model to represent the way in which schools' principals and teachers understand the concept of quality education. This model was built on the basis of qualitative opinions of teachers and principals from 12 schools. In order to organize the different components associated with quality education, the proposed model identifies three levels. The two most profound levels include components related to personal and emotional engagement with students, as well as values education and the development of soft skills, or 'life skills', while only the third, more superficial level, tackles the academic results and school's management processes. This model allows to better understand the way in which principals and teachers connect with the strategies for evaluating quality (mainly Simce), as well as other related educational policies.

Keywords: quality education, educational quality, quality evaluation, values education, school management, Simce.

Este artículo reporta los resultados de un estudio apoyado por el CNED, a través de su Convocatoria de apoyo a la investigación en educación 2014.

2 EDECSA (Consultora especializada en estudios educacionales). Contacto: pablotorche@ edecsaestudios.cl

3 EDECSA. Contacto. javimartineza@gmail.com

4 EDECSA. Contacto: jmadridsalazar@umail.ucsb.edu

5 EDECSA. Contacto: javier_araya@hotmail.com 
104 ¿QUÉ ES "EDUCACIÓN DE CALIDAD" PARA DIRECTORES Y DOCENTES? - P. Torche, J. Martínez, J. Madrid y J. Araya

\section{Antecedentes}

El concepto de educación de calidad o calidad educacional ha estado en el centro del debate público y académico en las últimas décadas en el país, poniendo de manifiesto visiones diversas, a veces contrapuestas, acerca de la forma en que se le define (ambos conceptos son utilizados de forma indistinta en el presente artículo). Mientras algunos enfatizan las condiciones de gestión de los establecimientos, otros ponen el foco prioritariamente en los resultados de aprendizaje, medidos a través del Simce, en tanto surgen crecientemente voces que abogan por un concepto más integral de calidad, que incluya también dimensiones como la valórico-espiritual, la artística y la deportiva, entre varias otras. No obstante, y quizás precisamente a causa del carácter controversial del concepto, la definición precisa de lo que se entiende por educación de calidad ha sido esquiva.

Los cuerpos legales, surgidos en los últimos años para mejorar la calidad de la educación, han hecho poco por despejar estas dudas. Así por ejemplo, la Ley Nacional de Aseguramiento de la Calidad de la Educación, SNAC, de 2011, establece en su Artículo 1 una definición general del proceso educativo, incluyendo el desarrollo "espiritual, ético, moral, afectivo, intelectual, artístico y físico, mediante la transmisión y el cultivo de valores, conocimientos y destrezas", pero no entrega una definición explícita de lo que se entenderá por educación de calidad en particular.

En términos operativos, si bien el proceso educativo es definido de forma amplia e integral, incorporando un conjunto de dimensiones, los mecanismos de evaluación establecidos tienden a orientarse casi exclusivamente a los resultados de aprendizaje, utilizando el Simce, como su principal indicador. En efecto, según el Artículo 18 de la ley, el proceso de la ordenación de los establecimientos, que en la práctica constituye una evaluación conducente a sanciones, deberá considerar los resultados del Simce con una ponderación mínima del $67 \%$, en tanto que los otros indicadores de calidad tendrán un máximo de 33\% de ponderación. Estos otros indicadores, según la definición de la Agencia de Calidad, incluyen: autoestima académica, clima y convivencia escolar, participación y formación ciudadana, hábitos 
de vida saludable, asistencia escolar, retención escolar, equidad de género y titulación técnico profesional ${ }^{6}$.

De esta forma, si bien se insinúa una definición amplia del proceso educativo, las estrategias de evaluación quedan referidas fundamentalmente a resultados de aprendizaje.

Así también, gran parte de las políticas educativas del Ministerio de Educación orientadas a mejorar la calidad, se articulan sobre la base de los resultados del Simce: el ordenamiento de los establecimientos, los planes de la Subvención Escolar Preferencial (SEP), la asignación docente, la clasificación del Sistema Nacional de Evaluación de Desempeño (SNED) y la información para padres. A pesar de que otras dimensiones de calidad son incorporadas en programas institucionales, estas raramente se asocian a consecuencias, o premios para los colegios.

Mientras las políticas públicas han privilegiado fuertemente los resultados de aprendizaje (medidos a través del Simce), como el principal parámetro para medir la calidad de la educación, el profesorado y diversos actores (véase por ejemplo Insunza, 2014) han manifestado recurrentemente sus críticas hacia una medición de la calidad que sería restrictiva e injusta, abogando por una evaluación más amplia. Recientemente, la crítica en particular al SIMCE, como un instrumento nocivo para evaluar la calidad de la educación, se ha extendido a diversos actores académicos y sociales agrupados por ejemplo en la plataforma Alto al Simce (alto-al-Simce.org).

Detrás de las críticas al Simce, se vislumbra una definición distinta del concepto de calidad educacional, más amplia y que considera otras dimensiones. De esta forma, el debate acerca de dicho instrumento de alguna forma sintetiza un cuestionamiento más amplio, por el concepto de educación de calidad que se prioriza a través de esta estrategia de evaluación.

En este contexto, resulta relevante preguntarse qué entienden los actores educativos de los establecimientos por educación de

6 Ver http://www.agenciaeducacion.cl/ordenacion/otros-indicadores-de-calidad-educativa/ 
106 ¿QUÉ ES "EDUCACIÓN DE CALIDAD" PARA DIRECTORES Y DOCENTES? - P. Torche, J. Martínez, J. Madrid y J. Araya

calidad, es decir, examinar en profundidad sus creencias, significados y valoraciones asociadas a este concepto. Se estima que este tipo de conocimiento será relevante para alimentar el diseño de políticas educativas más consistentes con la visión de directores y profesores $y$, en particular, hacer más efectiva su implementación a través de estrategias que dialoguen de forma más fluida con la realidad y las percepciones de aquellos encargados de ejecutarlas.

\subsection{La investigación acerca del concepto de calidad educacional desde la perspectiva de los actores educativos}

Curiosamente, es muy escasa la investigación especializada que se ha enfocado directamente en levantar la construcción subjetiva de los actores educativos respecto de un concepto tan determinante como es el de educación de calidad. En general, la investigación en torno al tema se ha centrado en identificar la asociación entre distintos factores de gestión escolar y los resultados de aprendizaje, lo que constituye una perspectiva completamente distinta. A partir de esta, más que comprender la calidad de la educación, lo que se quiere es determinar la eficiencia escolar de los centros educativos, buscando definir factores asociados al parámetro único de los resultados de aprendizaje.

Sin embargo, muy pocas investigaciones han tomado en cuenta la dimensión subjetiva de los actores educativos, es decir, qué es lo que los directores y docentes piensan que es una educación de calidad. De esta forma, las opiniones y significados de quienes imparten la educación han sido largamente desconsiderados.

La revisión bibliográfica permite constatar que no existe prácticamente ningún estudio o investigación formal que haya abordado directamente este objetivo. El ámbito de investigación que más se aproxima al tema, se enmarca en el concepto de "creencias de los profesores" (teachers' beliefs), pues aborda también la esfera subjetiva, es decir, las percepciones y construcciones mentales de los profesores respecto de distintos aspectos de su práctica. Estos estudios constatan que las creencias de los docentes sí influyen en su práctica educativa, confirmando así la interacción entre la esfera subjetiva de nociones, ideas y representaciones, con la esfera objetiva referida a la práctica docente. 
En este contexto, Griffiths, Gore J. \& Ladwig (2006) por ejemplo, encuentran que ciertas "creencias generales" de los profesores respecto de la pedagogía, sí se relacionan con la calidad de su práctica docente. Dos dimensiones en particular que se confirmaron como relevantes fueron las creencias en la propia capacidad y en la capacidad de los niños. El estudio sugiere también que este tipo de creencias más generales o fundamentales, se correlacionan de manera más clara con la calidad de la práctica pedagógica, que creencias o conocimientos más específicos respecto de ciertas metodologías o programas en particular.

Yero (2002) también encuentra que las creencias de los profesores afectan la forma en que estos se comportan. Si los profesores tienen confianza en un programa o metodología de enseñanza, y, sobre todo, si este programa es consistente con sus propias creencias sobre la educación tenderán a relevar aspectos positivos de dicho programa. En cambio, si sus creencias son contrarias al programa, tenderán a ver sus aspectos inefectivos. En la misma línea Calderhead (2006), estudiando la enseñanza de la escritura, encuentra que aspectos de la práctica docente como la forma en que los profesores preparan y organizan a sus niños, se vincula con sus apreciaciones mentales y también con la influencia de un determinado contexto.

Este tipo de estudios, no obstante, tiende a estar focalizado en creencias acerca de metodologías de enseñanza o prácticas educativas muy específicas, más que en conceptos amplios y abarcativos como el de educación de calidad.

En el caso chileno, muy pocos estudios han abordado la percepción de calidad de la educación desde los actores y solo lo han hecho tangencialmente. Carrasco (2014), en el marco de un estudio orientado a conocer las prácticas de selección de los establecimientos educacionales, aborda las creencias de los directores respecto de los factores que explicarían la buena reputación de sus establecimientos entre los padres: el 25,2\% considera que, en primer lugar, se encuentra el desempeño del colegio en pruebas estandarizadas, en tanto que un 22\% señala la formación valórica del establecimiento. Más abajo aparece la tradición $(16,8 \%)$ y el comportamiento de los alumnos $(11,2 \%)$. 
108 ¿QUÉ ES "EDUCACIÓN DE CALIDAD" PARA DIRECTORES Y DOCENTES? - P. Torche, J. Martínez, J. Madrid y J. Araya

Otro estudio, Raczynski (2011), orientado a describir el paso de alumnos de familias de estratos bajos de enseñanza básica a media concluye que para estas familias los resultados del Simce son irrelevantes, y que consideran otros indicadores de calidad para elegir establecimiento, entre los que se mencionan el nivel de disciplina, orden y seguridad, infraestructura y equipamiento, entre otros.

En tanto, en una encuesta realizada por la Universidad Diego Portales (2013), los directores revelan poseer un concepto más bien multidimensional de educación de calidad, que considera la dimensión académico-cognitiva, pero que incorpora también otras referidas al desarrollo emocional y afectivo y a la formación ciudadana. De esta forma, si bien el "logro académico/aprendizaje cognitivo" es la dimensión más importante, mencionado en primer lugar por un 53\% de los encuestados, convive con otras dimensiones también consideradas muy relevantes, como el desarrollo emocional y afectivo (mencionado en primer lugar por un 28\% de los encuestados) y la formación ciudadana y respeto a la diversidad (mencionado en primer lugar por un 14\%).

\subsection{La importancia de las creencias de los actores educativos para la implementación exitosa de políticas educativas}

Por último, interesa examinar aunque sea brevemente, la importancia de la esfera subjetiva de los actores educativos, en la implementación de políticas y programas educativos. En esta línea, existe bibliografía especializada que ha examinado en particular la forma en que profesores y otros actores responden a distintos tipos de reforma escolar. Datnow (1998) por ejemplo, ha encontrado grados de aceptación-resistencia variable dependiendo de las características de los docentes y su contexto de trabajo. En el mismo tema, Fullan (1991, 1993) establece que la receptividad de los docentes hacia los procesos de reformas, depende en gran medida de su nivel de involucramiento en el diseño y ejecución de los procesos de cambio, aspecto que es reforzado por Sarason (1996). Como resulta previsible, en general el cambio impuesto desde arriba y sin consulta genera reacciones de distancia, suspicacia y rechazo, en tanto que aquel 
que consulta la opinión de los profesores y es concordante con los objetivos de la escuela, recibe más grados de aceptación (Bailey, 2000; Sikes, 1992).

En una investigación más reciente, que se nutre de esta vertiente de la investigación especializada, Datnow y Castellano (2000) estudian en concreto la recepción de una reforma específica para la escuela en Inglaterra, denominada Éxito para Todos (EPT, en inglés, Success for All). Este programa está orientado a organizar los recursos para enfocarse en la prevención y en la intervención temprana, para asegurar que los estudiantes tengan éxito en lectura a lo largo de la primaria. Se examinó en concreto cómo los profesores respondieron al programa, y cómo sus creencias, experiencias y adaptaciones programáticas influenciaron su implementación.

Los resultados permitieron distinguir cuatro tipos de reacciones distintas entre los profesores, que van desde un fuerte apoyo a EPT hasta resistencia. Estas diferencias se encontraron en ambos establecimientos estudiados, y no dependían de características personales de los profesores. En general, las diferencias se asociaron con la percepción que los profesores tenían del programa y de cómo este se asociaba con sus creencias acerca de la educación y las metodologías de enseñanza. Los profesores que expresaban una resistencia más clara al programa tendían a pensar que este no era útil para ellos, y no se asociaba con la visión que tenían de los procesos educativos.

A partir de este tipo de estudios, se estima que el nivel subjetivo de percepciones y creencias de los actores educativos constituye un terreno fértil para estudiar y potenciar la implementación de distintos procesos de cambio y mejora en las escuelas. Dada la centralidad de un concepto como educación de calidad, la construcción de un modelo que explique de mejor forma la manera en que directores y docentes entienden este concepto y operan con él, puede otorgar luces útiles para estudiar la forma en que se relacionan con las distintas políticas orientadas a fortalecer esta calidad. 
110 ¿QUÉ ES "EDUCACIÓN DE CALIDAD" PARA DIRECTORES Y DOCENTES? - P. Torche, J. Martínez, J. Madrid y J. Araya

\section{Metodología}

Se utilizó una metodología de carácter cualitativo, tendiente a levantar y construir el concepto de educación de calidad desde la perspectiva de los directivos y docentes. Con este propósito se utilizó la metodología de la Grounded Theory (o teoría aterrizada), según ha sido formalizada por Glaser y Straus y, posteriormente, Straus y Corbin. Esta teoría fue concebida precisamente para levantar los conceptos, opiniones y discursos desde el terreno y provee procedimientos y técnicas sistematizadas de codificación y análisis para recoger de manera inductiva, conceptos, modelos y esquemas de pensamiento acerca de distintos aspectos de la realidad social.

\subsection{Técnicas de recolección de información}

Para la indagación del concepto de educación de calidad de directivos y docentes, se utilizaron grupos focales (focus groups) y entrevistas en profundidad, con el objeto de rescatar las visiones y discursos de los entrevistados, ofreciendo la posibilidad de indagar en profundidad en las representaciones, creencias y significados de los entrevistados, a través del examen de transcripciones textuales de las conversaciones.

Para los directores de los establecimientos se privilegió la realización de entrevistas individuales más que focus groups, para obtener la visión de cada director en relación con su contexto específico de trabajo. Asimismo, la entrevista individual con directores permitió rescatar de mejor forma su trayectoria personal y profesional. Para los profesores en cambio, se consideró más apropiada la técnica de focus groups, para recoger el discurso de un número mayor de docentes que representara, además, la enseñanza de distintos subsectores y distintos niveles. Para cada focus se resguardó que todos los profesores pertenecieran al mismo establecimiento, de tal forma de rescatar sus representaciones y percepciones de calidad, en vinculación con un contexto educacional específico. De esta forma, el concepto de educación de calidad levantado se relacionó siempre con la dinámica y cultura organizacional de cada establecimiento determinado. 


\subsection{Instrumentos de recolección de información y dimensiones de análisis}

Los focus y las entrevistas fueron realizadas a partir de pautas de algunas preguntas guía predefinidas. Ambas pautas fueron muy similares, para propiciar la transversalidad del análisis, introduciendo solo diferencias menores para ajustar a la realidad de cada cargo. La aplicación de las pautas fue muy libre, aceptando gran flexibilidad en su aplicación para ajustarse a la dinámica de la conversación, privilegiando siempre las prioridades y perspectivas de los entrevistados(as). En términos generales se abordaron los siguientes temas:

- Trayectoria personal y profesional: años de experiencia, motivación, satisfacciones y dificultades, logros y expectativas.

- Concepción de educación de calidad: definición de educación de calidad, dimensiones y jerarquización, objetivos, tipo de alumno y egresado ideal, escenas representativas de calidad.

- Percepción de las estrategias vigentes de evaluación de calidad: percepción y evaluación del Simce y otros sistemas de evaluación de calidad, aspectos priorizados y no considerados en esta, mecanismos ideales de evaluación de calidad, sugerencias.

\subsection{Diseño muestral}

Se realizaron 12 entrevistas con directores y 12 focus groups con profesores en 12 establecimientos de tres regiones del país. Para la selección de los casos se consideró como unidad muestral básica el tipo de establecimiento. Esta definición supone que la concepción de educación de calidad de los actores educativos indagados se puede relacionar de alguna manera con el tipo de contexto, cultura y organización educativa en la que están insertos.

Para la selección de los establecimientos se consideraron tres criterios básicos. En primer lugar el nivel socioeconómico, distinguiendo establecimientos de nivel bajo (definidos como A o B según clasificación Simce) y establecimientos de nivel medio y medio alto (definidos como C, D y E según la misma clasificación). En segundo lugar, se consideró también como criterio de segmentación el tipo de resultados Simce, que es actualmente el parámetro más 
112 ¿QUÉ ES "EDUCACIÓN DE CALIDAD" PARA DIRECTORES Y DOCENTES? - P. Torche, J. Martínez, J. Madrid y J. Araya

determinante para atribuir calidad, tomando como referencia la prueba de Matemáticas de $4^{\circ}$ y $8^{\circ}$ básico, y distinguiendo dos rangos de resultados:

- Bajos resultados: aproximadamente hasta 250 puntos en el Simce.

- Resultados medios y altos: Más de 250 puntos en el Simce.

En tercer lugar, se incorporó de manera exploratoria una variable de segmentación asociada al tipo de valoración que tiene el director respecto del Simce. De esta forma, se definieron los siguientes grupos de directores, atribuidos por el mismo entrevistador a partir de la información recopilada en la entrevista:

- Valora el Simce: aquellos directores que lo consideran un sistema válido y relevante para evaluar la calidad de la educación de un establecimiento.

- Indeciso o rechaza el Simce: aquellos directores que manifiestan un juicio abiertamente contrario al Simce, considerándolo poco válido, o bien mixto, indicando que tiene importancia pero también que presenta graves falencias.

En añadidura, se incluyeron también tres liceos con enseñanza Técnico Profesional (TP) y una escuela rural.

A partir de esta segmentación, se confeccionó una muestra de 12 establecimientos, según indica la siguiente tabla.

Tabla 1: Muestra final de establecimientos

\begin{tabular}{l|c|c|c|c|c}
\hline \multirow{2}{*}{} & \multicolumn{4}{|c|}{ Clasificación socioeconómica } & \multirow{2}{*}{ Total } \\
\cline { 2 - 5 } & $\begin{array}{c}\text { Nalora el } \\
\text { Simce }\end{array}$ & $\begin{array}{c}\text { Indeciso 0 } \\
\text { rechaza Simce }\end{array}$ & Valora el Simce & $\begin{array}{c}\text { Indeciso 0 } \\
\text { rechaza Simce }\end{array}$ & \\
\hline $\begin{array}{l}\text { Establecimientos } \\
\text { con bajos } \\
\text { resultados Simce }\end{array}$ & 2 & 1 & 2 & 1 & 6 \\
\hline $\begin{array}{l}\text { Establecimientos } \\
\text { con resultados } \\
\text { medios y yltos }\end{array}$ & 2 & 1 & 2 & 1 & 6 \\
\hline Total & 4 & 2 & 4 & 2 & 6 \\
\hline
\end{tabular}




\subsection{Estrategia de análisis}

El procedimiento de análisis de las entrevistas y focus se realizó sobre la base de transcripciones textuales, a través de un procedimiento de codificaciones sucesivas de carácter inductivo, de manera de avanzar hacia un nivel de abstracción cada vez mayor. La codificación según este sistema avanza de acuerdo con etapas, desde la codificación abierta de conceptos, muy apegada a los datos, hasta la codificación categórica y axial, que incorpora un trabajo interpretativo de los conceptos y categorías generadas, para establecer relaciones y jerarquizaciones que, aunque los entrevistados no hayan mencionado explícitamente, entreguen una representación fidedigna de su forma de comprender y construir los fenómenos de la realidad. De esta forma, se busca construir un modelo explicativo del concepto de educación de calidad que represente la concepción de los directores y docentes.

\section{Resultados}

\subsection{Dificultades para una definición}

El estudio permite distinguir en primer lugar un concepto amplio, complejo y en alguna medida heterogéneo de calidad educacional, donde directores y profesores introducen un conjunto variado de componentes, dando lugar a distintas prioridades y énfasis según sus visiones personales y el contexto en que se desempeñan.

Las dimensiones o componentes del concepto de educación de calidad son de muy distinta índole o nivel lógico. Algunos se relacionan con aspectos del compromiso y motivación personal del director(a) o docente, otros con los logros académicos y vitales de los alumnos, y otros con distintos elementos de la gestión escolar (construcción de comunidad, planificación y evaluación, capacitación docente y varios otros). Se distinguen también componentes de orden cognitivo, valórico y práctico en la formación de los estudiantes y, en menor medida, aspectos relacionados con las dimensiones artística y deportiva.

En este contexto, el estudio identifica en primer lugar una dificultad de los entrevistados para jerarquizar los distintos 
componentes que forman parte de una educación de calidad. Junto con esta dificultad para priorizar, los actores educativos manifiestan también una resistencia, muchas veces explícita, a articular estos componentes en un modelo organizado, que establezca categorías centrales y secundarias, o distintos ámbitos o áreas de significado o gestión. Para algunos, el mismo hecho de definir analíticamente una educación de calidad, desvirtúa su sentido y se manifiesta un escepticismo respecto de la posibilidad de arribar a una definición exhaustiva y coherente del fenómeno.

\subsection{Componentes de la educación de calidad}

En este contexto, el estudio permite identificar y sistematizar un conjunto muy amplio de conceptos, factores o dimensiones de trabajo y objetivos, de distinto orden, relativamente excluyentes entre sí, que aparecen en el discurso de directores y docentes explícitamente asociados con la idea de una educación de calidad. Es lo que se ha denominado, a un nivel completamente descriptivo, componentes de una educación de calidad. A pesar de que carecen de jerarquización o articulación entre sí, ofrecen una primera aproximación relativamente exhaustiva al fenómeno de estudio. 
Tabla 2: Componentes de una educación de calidad por directores y docentes

\begin{tabular}{|c|c|}
\hline Componente & Descripción / subcomponentes / indicadores \\
\hline $\begin{array}{l}\text { Preocupación personal/ } \\
\text { emocional por los alumnos (as) }\end{array}$ & $\begin{array}{l}\text { - Vínculo afectivo/entrega de cariño. } \\
\text { - Preocupación "paternal" por problemas personales de los } \\
\text { alumnos(as). }\end{array}$ \\
\hline Componentes valóricos & $\begin{array}{l}\text { - Que sean "buenas personas". } \\
\text { - Honestidad. } \\
\text { - Respeto. } \\
\text { - Solidaridad. } \\
\text { - Otros. }\end{array}$ \\
\hline $\begin{array}{l}\text { Aspectos formativos/formación } \\
\text { de hábitos para la vida }\end{array}$ & $\begin{array}{l}\text { - Esfuerzo, disposición al trabajo. } \\
\text { - Responsabilidad, puntualidad. } \\
\text { - Habilidades blandas: comunicación, trabajo en equipo, liderazgo }\end{array}$ \\
\hline $\begin{array}{l}\text { Educación "integral", } \\
\text { preocupación por la persona }\end{array}$ & $\begin{array}{l}\text { - Preocupación porque “sean felices”. } \\
\text { - Vínculo afectivo/entrega de cariño. }\end{array}$ \\
\hline $\begin{array}{l}\text { Logros académicos/resultados } \\
\text { de aprendizaje }\end{array}$ & $\begin{array}{l}\text { - Adquisición de conocimientos. } \\
\text { - Simce y pruebas relacionadas. } \\
\text { - Desarrollo de habilidades cognitivas. } \\
\text { - Ingreso a la universidad. }\end{array}$ \\
\hline Otros logros de los alumnos & $\begin{array}{l}\text { - Inserción laboral. } \\
\text { - Formar una familia. } \\
\text { - Ser feliz. } \\
\text { - Negativos (no caer en la delincuencia, no caer en la droga). }\end{array}$ \\
\hline Formación ciudadana & $\begin{array}{l}\text { - Aporte a la sociedad. } \\
\text { - Preocupación por los demás (solidaridad, altruismo). } \\
\text { - Participación democrática. }\end{array}$ \\
\hline Convivencia y disciplina & $\begin{array}{l}\text { - Buen ambiente y buenas relaciones. } \\
\text { - Buena disciplina. } \\
\text { - Bajo nivel de violencia, agresividad. } \\
\text { - Bajo nivel de bullying. }\end{array}$ \\
\hline Construcción de comunidad & $\begin{array}{l}\text { - Comprometer y motivar a los profesores. } \\
\text { - Promover el trabajo en equipo. } \\
\text { - Involucrar a los apoderados. }\end{array}$ \\
\hline Liderazgo directivo & $\begin{array}{l}\text { - Procesos de monitoreo de aula. } \\
\text { - Procesos de planificación. } \\
\text { - Construcción y reformulación del proyecto educativo. }\end{array}$ \\
\hline Gestión pedagógica & $\begin{array}{l}\text { - Capacitación y perfeccionamiento. } \\
\text { - Participación docente. } \\
\text { - Tiempo para la planificación. }\end{array}$ \\
\hline Formación deportiva & - Formación deportiva. \\
\hline Formación artística y cultural & $\begin{array}{l}\text { - Formación cultural. } \\
\text { - Talleres artísticos o creativos. } \\
\text { - Vinculación con manifestaciones artísticas o culturales. }\end{array}$ \\
\hline
\end{tabular}


116 ¿QUÉ ES "EDUCACIÓN DE CALIDAD" PARA DIRECTORES Y DOCENTES? - P. Torche, J. Martínez, J. Madrid y J. Araya

\subsection{Discursos acerca de la educación de calidad}

Más allá de los distintos componentes incluidos en el concepto de educación de calidad, el estudio permite identificar al menos tres discursos de carácter transversal, que organizan la visión de directores y docentes acerca de este fenómeno. Por discurso se entiende un conjunto de ideas articuladas explícitamente entre sí, y que los actores educativos utilizan consistentemente y de modo transversal, para construir el concepto de educación de calidad, y relacionarse con este desde su práctica cotidiana.

Estos discursos se refieren al carácter multidimensional de la educación de calidad, la referencia fundamental al contexto para poder definirla, y, por último, a la utilización de una metáfora familiar para abordar el tema.

a. El carácter multidimensional de la educación de calidad Los actores educativos se niegan a conceptualizar la educación de calidad en torno a un grupo reducido de ciertos componentes específicos. Incluso, como se ha mencionado, jerarquizar los componentes de una educación de calidad resulta dificultoso, porque desde su perspectiva ningún componente adquiriría prioridad sobre otro. La educación de calidad sería esencialmente una condensación de un conjunto de factores, que no pueden separarse entre sí, algo que los directores y docentes aluden frecuentemente con la denominación de educación integral o integralidad.

El fondo de este discurso parece referir a la idea de que una educación de calidad requiere de una aproximación esencialmente holística por parte de sus actores y, por tanto, el análisis o fragmentación de la misma reduce su valor o la desvirtúa de alguna forma. Por esta razón, la aproximación al trabajo educativo, desde una lógica de procesos de gestión separados - usual en el diseño de las políticas educativas-, produce desconfianza o rechazo en algunos actores educativos, que consideran que no captura adecuadamente la esencia de una educación de calidad y, por ende, no es adecuada para promoverla y evaluarla. 
Dentro de esta multidimensionalidad o carácter integral de la educación de calidad, los actores educativos hacen énfasis también en que esta no se restringe puramente a la dimensión académica, deslizando una crítica a la excesiva importancia que se otorga a esta dimensión desde las distintas políticas educativas de refuerzo y evaluación.

(...) o sea hay varios elementos más que forman parte de esta "integralidad", (...) al igual que el cuerpo humano que está compuesto de distintos órganos, la educación es igual, entonces toda vez que se entregan elementos de la formación... la formación valórica general, la formación de pastoral, se da la posibilidad que los estudiantes exploren otras experiencias a nivel más artístico, más deportivo, a través de academias o talleres extracurriculares, también se les abre la posibilidad de una educación que sea más integral, que lo llevan más allá de la sala de clases. (Profesor, particular sub., GSE medio alto, Simce medio alto).

b. El carácter contextual del concepto de educación de calidad Según los actores entrevistados la educación de calidad debe comprenderse como un concepto esencialmente dinámico y cambiante, que no puede definirse de manera fija, sino que debe ajustarse a las condiciones contextuales de los establecimientos. No sería posible, en consecuencia, hablar de una sola educación de calidad, sino de varios tipos de educación de calidad, dependiendo del contexto en el que se inserta.

Según los entrevistados hay dos criterios relevantes para definir el contexto en el que se inserta la educación de calidad. El primero de ellos, y por lejos el más importante, se refiere a las condiciones socioeconómicas de los estudiantes y sus familias. Relacionado con lo anterior, pero de alguna forma distinguible, se menciona también un segundo criterio, asociado a las características culturales del entorno, en especial en relación con contextos rurales.

A partir de estos criterios, los actores educativos construyen lo que podría denominarse un contexto de vulnerabilidad socioeconómica, que reclamaría un concepto específico de educación de educación calidad. 
118 ¿QUÉ ES "EDUCACIÓN DE CALIDAD" PARA DIRECTORES Y DOCENTES? - P. Torche, J. Martínez, J. Madrid y J. Araya

Es interesante destacar, no obstante, que prácticamente todos los actores entrevistados consideran que se desenvuelven en un contexto de vulnerabilidad, incluyendo los de algunos establecimientos de nivel medio y medio alto. En este sentido, el discurso en torno a una educación que se ajuste a contextos de vulnerabilidad, más que aludir a un contexto excepcional, podría reflejar más bien la demanda por ajustar a la realidad un concepto de educación de calidad que parece haber sido construido sobre condiciones excepcionales o de élite, que resultan ajenas al común de los colegios del país.

Yo creo que cada institución tiene una realidad distinta, tanto en el aspecto socioeconómico, sociocultural (...) por lo tanto lo que necesitan (...) no es lo mismo; la educación de calidad para un niño que vive en una ciudad como Santiago (es distinta) del que vive en una zona rural en la Patagonia. Sus "aprendizajes significativos" obviamente que están en un contexto distinto y por lo tanto lo que ese niño allá necesita no es lo mismo que necesita acá. (Profesor, particular sub., GSE medio alto, Simce medio alto).

Al hacer mención a un contexto de vulnerabilidad, los actores educativos aluden principalmente a lo que consideran una serie de falencias en la educación familiar, lo que genera responsabilidades extra por parte del establecimiento. De esta forma, en un contexto de vulnerabilidad, la educación de calidad debe hacerse cargo de más cosas que en un contexto no vulnerable o, como lo pone un profesor, debe "empezar de más abajo".

En un contexto de vulnerabilidad, la educación de calidad debe hacerse cargo, según los entrevistados, de tres aspectos fundamentales. En primer lugar, se mencionan elementos relacionados con las necesidades básicas y el cuidado personal: alimentación, vestuario y limpieza personal, entre otras. En segundo lugar, la preocupación personal y afectiva por los estudiantes adquiere una relevancia central, en un contexto que es percibido como de gran precariedad y carencia. Los actores educativos consideran que deben hacerse cargo de necesidades psicológicas de los alumnos, a partir de los conflictos familiares que pueden incluir problemas de violencia, maltrato e incluso abuso. En tercer lugar, se menciona una serie de 
normas básicas de conducta e interrelación personal (puntualidad, permiso para hablar, respeto por los demás, entre otras), que los(as) alumnos(as) no poseerían. En esta línea, la formación de valores y habilidades para la vida adquiere una relevancia fundamental, pues es considerada imprescindible para que los alumnos puedan desarrollar una vida satisfactoria, e integrarse a la sociedad.

Que de repente ayudar a los chiquillos a salir del ambiente tóxico en el que están porque el papá, el abuelo, la tía, el (entorno) familiar completo está metido en la droga o en la delincuencia y ojalá que el mismo cabro no repita las mismas prácticas. (Profesor, particular subvencionado, GSE bajo, Simce medio alto).

De repente ellos tienen una serie de problemáticas que no es porque el cabro no sepa, también hay una problemática social de por medio: muchos trabajan, se acuestan tarde, algunos se quedan chateando, etc. y tantos problemas, no hay una familia presente que esté colocando una disciplina, reglas. Ahora los papás han perdido autoridad entre sus hijos. Nosotros dentro de todo tratamos de entregar una educación de calidad. (...). Por ejemplo, el otro día hablábamos del caballo de Troya y había algunos que no sabían qué era el caballo de Troya, cosa que todo el mundo habla... hasta un niño chico sabe y ellos no sabían, entonces uno dice ¿por qué este niño nunca ha leído ni ha visto una película? Entonces yo, en vez de quejarme, voy a tener que contarle todo el caballo de Troya como cuento. (Profesor, municipal, GSE medio alto, Simce medio alto).

En el contexto de vulnerabilidad, los actores educativos suelen observar a las familias de los alumnos, más que como un aporte, como un obstáculo para los objetivos educacionales que persiguen. En este sentido se hace énfasis en el escaso capital cultural que poseen, las dificultades y desinterés para apoyar los procesos educativos de sus hijos y, sobre todo, la presencia de hábitos y pautas de relacionamiento que dificultan la educación, tales como falta de disciplina, conductas agresivas o, en casos más graves, valores contraculturales, consumo de drogas y vinculación con la delincuencia, entre otros.

Como la gran mayoría de los actores educativos, según se ha mencionado, considera que se desenvuelve en contextos de 
120 ¿QUÉ ES "EDUCACIÓN DE CALIDAD" PARA DIRECTORES Y DOCENTES? - P. Torche, J. Martínez, J. Madrid y J. Araya

vulnerabilidad, esta percepción puede adquirir la forma de una crítica más general a la pertinencia y relevancia del currículo, que debería ser estudiada en mayor profundidad.

c. La educación de calidad desde la metáfora familiar

Por último, se consigna un discurso transversal que tiende a concebir la educación de calidad principalmente en términos de dinámicas familiares, más que productivas o puramente profesionales. En esta línea, el establecimiento y sus procesos educativos no se comprenden como una organización funcional, sino más bien familiar, articulada principalmente en torno al afecto, el amor y la preocupación personal por todos sus miembros, en particular por sus alumnos. De esta forma, los directores, y sobre todo los docentes hacen énfasis una y otra vez en el establecimiento de un vínculo casi filial con sus alumnos, que resulta imprescindible para lograr una educación de calidad.

Este discurso es más explícitamente articulado en establecimientos que atienden alumnado vulnerable, pero llama la atención que, de una forma u otra, aparece en los actores de todos los establecimientos.

Esta metáfora familiar para concebir la educación de calidad refuerza el énfasis en los aspectos formativos, incluyendo la dimensión valórica y de habilidades para la vida, además de otorgar más fuerza a la dimensión personal o filial del vínculo entre el profesor y el alumno.

En este mismo contexto, la incorporación de nociones propias de la gestión instrumental, como objetivos, planificación y evaluación resultan ajenas para algunos actores y poco conectadas con su visión de una educación de calidad.

Imagínate que en enseñanza básica los chiquillos se ven desposeídos del amor maternal, fraternal o de algún familiar. Imagínate con lo que nos enfrentamos nosotros en enseñanza media. Ayer sin ir más lejos, una alumna de cuarto medio, se me puso a llorar y me dice: "iestoy mal!", "pero ¿qué te pasa?" -le dije. "Mi mamá no me quiere, mi mamá se juntó con su pareja, tuvo un hijo -me dijo- jtía!, es que yo hago de todo para que mi mamá me acepte", y lloraba a mares (...). 
Te encontrai con ese tipo de alumnos, que lograi sacarle algo positivo, lograi hacerlo pensar o suplirle esa falta de cariño que tiene. (Profesor, municipal, GSE medio alto, Simce medio alto).

Son cabros... ponte tú que llegan a su casa a preparar comida, acá no solamente eres profesora, eres psicóloga, socióloga, eres mamá, los varones son papás, consejeros... las tenís que hacer todas. (Profesor, municipal, GSE medio alto, Simce medio alto).

3.4. Modelo del concepto de educación de calidad desde la perspectiva de directivos y docentes

A pesar de que los mismos entrevistados se resisten a jerarquizar los distintos componentes que forman parte de una educación de calidad, a través del análisis de su discurso es posible detectar que no todos estos componentes tienen para ellos el mismo nivel de profundidad, ni guardan la misma relevancia. De esta forma, aunque los actores educativos no los organicen explícitamente en un modelo de prioridades, a través del análisis es posible identificar un ordenamiento transversal que organiza los diversos componentes de una educación de calidad, en tres niveles distintos.

El criterio articulador básico de este modelo se relaciona con el nivel de profundidad y relevancia asignado por los actores educativos a los distintos componentes que forman parte de la educación de calidad. A partir de este criterio, se diferencian aquellos que adquieren sentido por sí mismos, o bien, se relacionan con creencias y significados profundos de los entrevistados en relación con el proceso educativo de otros asociados con objetivos instrumentales o totalmente externos, pero que no se relacionan con sus propias creencias al respecto. A partir de este criterio, se distinguen tres niveles fundamentales para organizar los componentes de una educación de calidad, y que constituyen la primera aproximación a un modelo tentativo del concepto en estudio. Estos tres niveles, denominados, nivel esencial, nivel formativo y nivel instrumental, se describen a continuación, indicando también los componentes que incluye cada uno. 
122 ¿QUÉ ES "EDUCACIÓN DE CALIDAD" PARA DIRECTORES Y DOCENTES? - P. Torche, J. Martínez, J. Madrid y J. Araya

\section{a. Nivel esencial}

El primer nivel se ha denominado nivel esencial, puesto que incluye componentes que adquieren valor y relevancia para los entrevistados exclusivamente a partir de sus creencias y significados asociados al proceso educativo, sin que sean necesarios objetivos funcionales, ni presiones externas para justificar su relevancia.

Los componentes del nivel esencial comprometen el significado más inmediato de la acción educativa, que se juega en la relación personal y humana con los alumnos, más allá de cualquier consideración formativa o instrumental. Se trata de componentes que destacan el aquí y el ahora de la práctica educativa, el presente por sobre el cumplimiento de objetivos a futuro, y que se centra en que el niño sea feliz, esté motivado y asista al colegio con ganas.

El componente esencial que se incluye en este nivel se refiere a la construcción de un vínculo y compromiso personal con los alumnos(as). Dentro de este componente se mencionan aspectos como la entrega de afecto y amor (preocupación afectiva), el cuidado de aspectos psicológicos y también la preocupación por necesidades básicas no cubiertas (como alimentación o vestuario). Este componente se inscribe por lo general en la metáfora familiar ya mencionada, según la cual los directores y profesores establecen con los alumnos un vínculo de tipo filial. El proceso educativo no tiene sentido para ellos sin este trasfondo familiar y, por lo tanto, tampoco se puede pensar en una educación de calidad sin este. Prácticamente para todos los directores y docentes entrevistados, este componente constituye el fundamento para cualquier proceso de educación de calidad.

Pero el trabajo en el aula es muy gratificante para mí, yo lo hago con mucho cariño, con amor también, porque yo creo que por ahí también pasan los cambios, de la forma que uno enseña con amor y con afecto, creo que eso es muy importante y Gabriela Mistral lo decía "enseñar con amor" ¿me entiendes? Y eso es fundamental en el trabajo que uno hace en estos días, en el trabajo que uno realiza. (Profesor, municipal, GSE medio alto, Simce medio alto). 


\section{b. Nivel formativo}

El segundo nivel, denominado formativo, incluye componentes que se asocian con las creencias y significados profundos de los actores en relación con el proceso educativo, pero guardan también una clara dimensión funcional, en el sentido de que se orientan también al cumplimiento de objetivos que serán importantes para el desarrollo futuro de los(as) alumnos(as).

De esta forma, los componentes de este nivel se fundan en una visión propia del ser humano, pero se justifican y adquieren mayor importancia sobre la base de la utilidad que tendrán para la inserción y desempeño exitoso de los alumnos en la sociedad. Su relevancia por tanto no se encuentra solo en una creencia o sentido antropológico del director o profesor, sino también en la utilidad o efecto formativo que tendrán para el futuro.

Se incluyen aquí fundamentalmente dos componentes. En primer lugar, la formación valórica de los alumnos, asociada principalmente con la aspiración de los educadores de formar buenas personas, honestas y solidarias (entre otros valores). Un segundo componente, relacionado pero diferenciable, se relaciona con la formación de habilidades para la vida, que incluye hábitos que directores y docentes consideran cruciales para la vida de sus alumnos, tales como responsabilidad, esfuerzo, capacidades comunicativas y otros.

Ambos componentes son considerados cruciales para el desarrollo futuro de los alumnos en relación con objetivos como conseguir trabajo, formar una familia e integrarse adecuadamente a la sociedad. Es importante destacar que estos aspectos pueden adquirir más preponderancia en establecimientos que atienden a una población vulnerable, aun cuando forman parte del discurso de actores educativos que trabajan con todo tipo de alumnado.

Poder entregar más allá de que los alumnos aprendan matemática o lenguaje, es poder enseñarles un poco a vivir. (Profesor, particular subv., GSE medio alto, Simce bajo). 
Poder entregarle conocimiento como para que puedan desarrollarse en la vida, con valores, con principios (...) siento que eso a las personas les deja mucho más. Yo actualmente tengo exalumnos que son profesionales en muchas áreas y qué lindo cuando a uno le dicen "profesora sabe que tengo tan buenos recuerdos, qué rico haberme encontrado con usted" (...) qué bueno porque no creo que ellos se acuerden de lo que yo les enseñé en matemática. (Profesor, particular subv., GSE medio alto, Simce bajo).

El concepto de calidad de educación, para mí, es que seamos capaces de formar personas integrales, ciudadanos de este país ¿ya?, conscientes de sus deberes y obligaciones y capaces de poder insertarse a futuro en los niveles de educación que decidan, inicialmente la educación secundaria, científico humanista, técnico profesional. (Director, particular subv., GSE bajo, Simce bajo).

\section{c. Nivel de instrumental, de gestión}

Por último, el tercer nivel se ha denominado instrumental, ya que no adquiere justificación a partir de las creencias o motivaciones intrínsecas de los actores educativos, sino en relación con los objetivos por lograr a través del proceso educativo, así como con lineamientos y regulaciones externas.

Se incluyen aquí aquellos componentes de una educación de calidad que, si bien son mencionados con mucha frecuencia por directores y docentes, no se conectan directamente con sus creencias, significados y motivaciones más profundos en relación con una educación de calidad.

Participan principalmente dos tipos de componentes. En primer lugar, aquellos relacionados con la formación académica y los logros de aprendizaje, que aparecen como un parámetro transversal de calidad mencionado por todos los entrevistados con mayor o menor importancia. En este ámbito se identifican de manera concreta la adquisición de aprendizajes fundamentales y el desarrollo de habilidades cognitivas. Así también, el Simce, el puntaje PSU y el ingreso a la universidad aparecen reiteradamente como indicadores relevantes de logro. 
Yo visualizando también los resultados que he obtenido con mis cursos anteriores, como cuando con el segundo básico construimos el puntaje más alto de los colegios municipales en el Simce, así que esa imagen va a quedar en el tiempo y uno hace preguntas de eso y los niños lo han retenido, entonces uno ahí se ha dado cuenta de que han aprendido ese contenido como tal. (Profesora, municipal, GSE medio alto, Simce medio alto).

En segundo lugar, se puede incluir también aquí un conjunto de componentes relacionados con la gestión escolar. Se hace mención a diversos procesos de gestión, planificación y evaluación, asociados con temas como la construcción de comunidad, la cobertura curricular, el involucramiento de los apoderados y el manejo de la convivencia, entre otros. En términos más concretos, múltiples directores hacen referencia a elementos relacionados con la formación y capacitación docente, y la necesidad de contar con más tiempo e instancias para planificar y reflexionar en torno a la práctica pedagógica.

(...) pero nos falta un aspecto importante que (son los) logros educativos, que nuestros niños logren estar en la media nacional de los estándares, de los estándares de aprendizaje. Es decir, que nuestros niños tuvieran en las pruebas estandarizadas sea Simce u otros (...) 250 puntos, pero nosotros andamos por ahí por los 230. (Director, municipal, GSE bajo, Simce bajo).

\section{d. Determinantes y contexto}

Por último, para construir un modelo del concepto de educación de calidad que mantienen los actores educativos, resulta imprescindible incluir la dimensión contextual del mismo, es decir, aquellos factores que, si bien no forman parte del núcleo de una educación como tal, sí determinan de modo importante la posibilidad de conseguirla.

Se distinguen aquí condiciones contextuales y determinantes. Las primeras, se relacionan principalmente con las características socioeconómicas y culturales de los alumnos y sus familias. Como se ha mencionado, según los entrevistados estas condiciones no solo afectan las posibilidades de alcanzar una educación de calidad, sino que ejercen una influencia más profunda, en el sentido de definir y 
126 ¿QUÉ ES "EDUCACIÓN DE CALIDAD" PARA DIRECTORES Y DOCENTES? - P. Torche, J. Martínez, J. Madrid y J. Araya

moldear la forma misma en que se debería definir la educación de calidad.

Junto con esta influencia contextual más amplia, los actores educativos establecen una serie de determinantes relevantes de la educación de calidad, que constituyen factores más específicos que posibilitan o dificultan su implementación. Los actores educativos incluyen en este ámbito tres elementos fundamentales. El primero es el nivel de involucramiento de los apoderados, tanto en las actividades educativas del establecimiento como de manera general en el proceso educativo de sus hijos. En segundo lugar se hace mucho énfasis en la formación y perfeccionamiento docente, emergiendo un conjunto de críticas al nivel de las competencias de los profesores el cual, según los directores, actúa como un "techo" para la calidad. Por último, se mencionan también como un determinante relevante las condiciones de trabajo en que se desenvuelven los profesores, haciendo énfasis en los tiempos de planificación con que cuentan (lo que se conoce por horas "no lectivas") y la existencia de instancias de reflexión y aprendizaje al interior de los establecimientos. También se mencionan en este ámbito el nivel de los salarios, las condiciones labores y las características de la infraestructura del establecimiento.

Para lograr educación de calidad hay que desagobiar al profesor. Un profesor que tiene 44 horas frente a curso, con jornada escolar completa, que atiende 12 o 13 cursos y tiene que corregir pruebas y más de 100 alumnos que debe atender por semana, eso es agobio y locura. Pedagogía es una de las carreras con más deserción y licencias por estrés. Hay que disminuir (la cantidad de) alumnos por clase. No hay calidad, si los colegios no tienen que claro qué tienen que hacer. Yo creo que nos deben apoyar, en cuanto al profesor, los alumnos por curso, remuneración al docente, fiscalizar más. (Director, particular subv., GSE bajo, Simce medio alto).

A partir de esta distinción preliminar de tres niveles, que permitiría organizar y priorizar los distintos componentes levantados, así como de los condicionantes contextuales, es posible proponer un primer modelo tentativo del concepto de educación de calidad, que se expresaría en el siguiente diagrama. 
Diagrama 1: Modelo de educación de calidad desde la perspectiva de directores y docentes

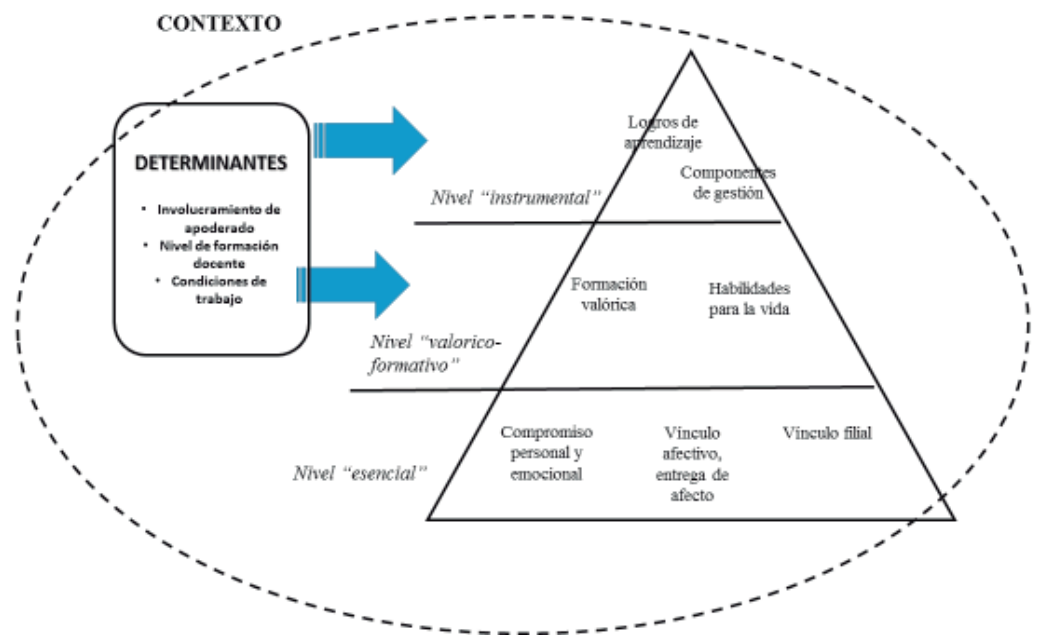

3.5. Percepción de las estrategias de evaluación de calidad

Los directores y docentes asocian el sistema o estrategias de evaluación de la calidad de la educación principalmente con el Simce. Otros mecanismos de evaluación -por ejemplo la evaluación de desempeño de la Agencia de Calidad y los otros indicadores de calidad (OIC)-, no han sido internalizados de modo transversal como componentes cruciales de un sistema de evaluación de calidad, y aparecen solo tangencialmente en el discurso de los entrevistados.

De esta forma, el Simce ocupa un rol crucial en las representaciones de los directores y docentes, a la hora de construir su visión del discurso de la institucionalidad pública en torno al concepto de educación de calidad. En relación con este, se recoge un discurso preponderantemente crítico, asociándoselo a una estrategia de evaluación de calidad estrecha e injusta. No obstante, también se detecta una serie de consideraciones positivas del Simce, que se presentan en primer lugar. A continuación, se desarrollan las razones por las cuales el SIMCE es evaluado tan negativamente, a la luz del modelo de calidad propuesto. 
128 ¿QUÉ ES "EDUCACIÓN DE CALIDAD" PARA DIRECTORES Y DOCENTES? - P. Torche, J. Martínez, J. Madrid y J. Araya

a. Aspectos valorados del Simce

La valoración positiva del Simce se produce principalmente en relación con la utilidad que presta un sistema de evaluación externo para orientar el trabajo de los establecimientos. Se valora aquí la utilidad de los resultados para el diagnóstico, identificando avances y rezagos. Los actores educativos que valoran el Simce tienden a sugerir un uso del mismo que no se orienta a establecer comparaciones entre establecimientos, sino a potenciar su apoyo. Esta visión es bastante transversal a los distintos tipos de establecimiento, incluidos aquellos que obtienen buenos resultados.

A mí no me molesta el Simce, no, no me molesta. Me parece que es bueno tener algo externo que te mida porque te hace sentir esa adrenalina de ser mejor, ahí está la competencia, pero sana porque ahí compites con el colegio, con tus propios resultados. (Directora, particular subv., GSE bajo, Simce medio alto).

De esta forma, directores y docentes tienden a recomendar un uso del Simce que no se oriente a establecer comparaciones entre los establecimientos, sino a apoyar y fomentar la comparación y mejora interna.

b. Críticas al Simce

A la luz del modelo de la concepción que directores y docentes tienen de la educación de calidad presentado más arriba, es posible comprender en mayor profundidad la percepción principalmente crítica que tienen los actores educativos respecto del Simce. En efecto, si se contrapone el Simce en relación con el modelo propuesto, se observa que este sistema de evaluación aborda únicamente aspectos relacionados con el nivel instrumental. Esto quiere decir que la parte más importante de lo que los actores educativos consideran como educación de calidad (el nivel esencial y formativo), no resulta evaluada por el Simce.

Los entrevistados configuran esta preocupación de modo fragmentario y no plenamente articulado en su discurso. Si bien reconocen que el Simce es necesario y presta una utilidad, resienten con fuerza el grado en el cual se invisibiliza parte importante de sus 
esfuerzos, desconsiderando precisamente aquellos componentes que ellos consideran más relevantes para lograr una educación de calidad. En la medida en que el Simce sirve de base para articular los discursos y estrategias de la política educativa, se transforma en el mecanismo que sintetiza para ellos la tensión entre la macroinstitucionalidad pública, y el trabajo que se desarrolla en los establecimientos, y evidencia las dificultades parar establecer un diálogo entre ambos.

A partir de la crítica al Simce, emerge también una fuerte demanda por reconocimiento, según la cual los actores educativos reclaman mecanismos de evaluación y valoración relacionados con aquellas dimensiones y componentes de su trabajo que se asocian con su visión de una educación de calidad. Esta demanda es mucho más clara entre los actores educativos que se desenvuelven en contextos vulnerables y en los profesores por sobre los directores.

En contrapartida, la desconsideración o invisibilización por parte del Simce de los componentes del nivel esencial y valórico es percibida por los actores educativos como una forma de violencia simbólica, que deniega sus esfuerzos, y que intenta imponer una visión de educación de calidad incongruente con sus convicciones más profundas. Esta sensación se expresa en diversos grados de molestia, frustración y sensación de abuso.

Aquí en Chile lamentablemente la calidad se ve con los resultados, entonces no se ve calidad de cualidad sino de cantidad, digamos, cuánto es lo que tú sabes, lo que no sabes. Ahora, el Simce y otras pruebas que son de medición (...) yo creo que la calidad de la educación tiene que ver incluso con el respeto, de enseñar a ser buenos ciudadanos, respetuosos, tener empatía con los demás, participar en equipo, trabajar en equipo, ser solidario. (Profesor, particular subv., GSE bajo, Simce medio alto).

Yo creo que la evaluación de la calidad tiene que ser una evaluación totalmente cualitativa si queremos ver si son alumnos integrales, porque por ejemplo un instrumento como el Simce, cierto, no demuestra si realmente la educación es de calidad porque no estamos viendo si el estudiante que estamos viendo es integral, es un complemento de varias áreas entre la parte, tal vez, conocimiento, la parte emocional, la parte 
como individuo ¿cierto?, como ciudadano incluso. Entonces no estamos viendo si es, como decir, un aporte a la sociedad, no es integral. (Profesor, particular subv., GSE bajo, Simce medio alto).

Esta tensión se agudiza en el caso de los actores educativos que trabajan en contextos de vulnerabilidad que, como se ha mencionado, tienden a valorar principalmente aquellas áreas de una educación de calidad que no son consideradas por el Simce. Para estos entrevistados, el Simce es incapaz de capturar y considerar elementos correspondientes al contexto, lo cual refuerza una sensación de injusticia y abuso, por cuanto se considera que el instrumento provee parámetros de comparación injustos, al comparar establecimientos con contextos y tipos de alumnados muy distintos. De esta forma, se critica en particular el uso del Simce que conduce, según los entrevistados, a la estigmatización y la estratificación.

(...) (tenemos alumnos en) situación de calle, papás privados de libertad, yo tengo alumnos en séptimo privados de libertad, drogadictos, etc. Entonces por eso acá, una de las cosas de la que hablaba es el tema sentimental, el tema emotivo (...) nosotros tenemos que trabajar con eso, modificarlo, que entregar cariño... a una profe le pegan, pero al cabro chico igual hay que seguirle dando cariño, porque el cabro chico está tirado en la casa... cómo evaluamos eso que está dentro de una educación de calidad. (Profesor, municipal, GSE bajo, Simce bajo).

(...) el número del Simce.te "estratifica" y te dice: ustedes son malos o son buenos, yo tengo súper claro por qué estamos en el número que estamos ¿ya?, pero ¿cómo salgo de ahí? solo con los recursos que tenemos estamos tratando de hacerlo. (Director, particular subv., GSE bajo, Simce bajo).

\section{Conclusiones y discusión}

A partir del estudio, se construye un modelo provisorio de educación de calidad, articulado en torno a tres niveles, que permite arrojar luz sobre la forma en que directores y profesores se enfrentan a este concepto, definen sus prácticas para alcanzarla y observan las estrategias de evaluación y apoyo de la calidad. 
El modelo propuesto se construye sobre la base de un primer nivel denominado esencial, que sirve de pilar para la construcción subjetiva en torno al tema, e incluye fundamentalmente el compromiso y la preocupación personal por los estudiantes. El segundo nivel, denominado formativo, incluye la entrega de valores y de habilidades para la vida. El tercer nivel, por su parte, denominado instrumental, incluye los logros de aprendizaje y los componentes de la gestión escolar.

A partir de este modelo es posible constatar una gran distancia entre la forma en que los directores y profesores conciben la educación de calidad, y la manera en que esta es conceptualizada y evaluada desde las instituciones educativas del Estado, distancia que es evidenciada por parte de los actores educativos principalmente en torno a la implementación y uso que se hace del Simce.

En efecto, de los niveles definidos, solo el instrumental (logros de aprendizaje y procesos de gestión escolar), parece recibir una atención más sistemática por parte de esta estrategia de evaluación. En tanto, el nivel esencial y el nivel formativo-valórico, que constituyen la base de la educación de calidad para directores y docentes, no recibe atención directa por parte de las estrategias de evaluación, y tampoco perciben que sea considerada de manera relevante por las estrategias de orientación y apoyo.

Esta desalineación es percibida de manera muy concreta por parte de los actores educativos entrevistados. En esta línea, el estudio detecta, en primer lugar, un problema de reconocimiento, con una fuerte demanda de valoración por parte de los actores entrevistados, que reportan además una sensación de maltrato y abuso. Por otro lado, se demandan también de manera directa estrategias de evaluación de la calidad que incorporen los aspectos que para ellos resultan más relevantes de una educación de calidad.

En este contexto, el estudio permite identificar también un fuerte grado de desacreditación del Simce como estrategia de evaluación de calidad, lo que permea también hacia la visión que directores y docentes construyen de las instituciones educativas. 
132 ¿QUÉ ES "EDUCACIÓN DE CALIDAD" PARA DIRECTORES Y DOCENTES? - P. Torche, J. Martínez, J. Madrid y J. Araya

A partir de estos resultados es posible hipotetizar respecto de la influencia que puede tener la dimensión subjetiva del concepto de educación de calidad, en la percepción y apropiación que hacen los actores educativos de los programas y políticas orientadas a fortalecerla. Si los establecimientos trabajan con un concepto de educación de calidad distinto al propuesto por la institucionalidad educativa, es poco probable que la apropiación y efectividad de los programas de apoyo a la calidad sean muy altas. Asimismo, resulta razonable estimar un grado importante de cumplimiento de funciones con un carácter puramente administrativo, pero sin una apropiación de fondo de las lógicas y los objetivos de las distintas estrategias de apoyo a la calidad.

Este tipo de procedimientos se detecta en el estudio, en particular en relación con las estrategias de preparación y adiestramiento para el Simce. En muchos establecimientos este tipo de estrategias es implementada y el Simce es referido como un estándar de logro, pero no porque se conecte con las creencias internas del director y menos con las de los profesores. Se trata más bien de un trabajo orientado a obtener una validación externa, lo que puede profundizar la tendencia a orientar la práctica educativa a partir de regulaciones heterónomas en vez de autónomas.

En particular en relación con estrategias asociadas al liderazgo educativo y a la gestión escolar, el estudio sugiere un grado variable de compromiso y motivación real. En concreto, no se percibe en el discurso de los entrevistados ninguna conexión entre las estrategias asociadas a una educación de calidad (planificación, monitoreo y evaluación de procesos), y aquellas dimensiones más valoradas de una educación de calidad. Por el contrario, parecen compartimentos estancos.

En este sentido, un obstáculo relevante para una mejor implementación de este tipo de procesos podría estar relacionado con una concepción de educación de calidad que no los valida como herramientas útiles para alcanzarla.

Para explorar en mayor detalle estas posibles consecuencias y estudiar también vías de solución, será necesario en primer lugar, 
validar el modelo construido. En este sentido, es necesario destacar que el presente estudio constituye solo una primera aproximación en un terreno ampliamente inexplorado, relacionado con la construcción subjetiva de los actores educativos acerca del concepto de la educación calidad. De esta forma, sería necesario en primer lugar contrastar el modelo construido con los mismos actores educativos, de tal forma de determinar si les hace sentido, y si consideran que refleja su visión de la educación de calidad. En la misma línea, resulta necesario también abordar cuantitativamente los aspectos vinculados con la percepción subjetiva de la educación de calidad, para mensurar el peso y prioridad asignada a las distintas dimensiones establecidas, así como el grado en que las características de los actores, o los establecimientos inciden en esta percepción.

En esta línea, sería interesante también estudiar en mayor profundidad en qué medida un modelo de este tipo puede servir para configurar distintas tipologías de directores y profesores. En efecto, el estudio permite visualizar distintos grados en que tanto el discurso como las políticas promovidas por la institucionalidad central son apropiados por los actores educativos. De esta forma, se detectan algunos actores educativos que parecen haber hecho suyo de mayor manera el lenguaje y las lógicas de la política educativa, en tanto que otros se muestran particularmente resistentes a ellas, lo que podría estar relacionado con distintas formas de concebir la educación de calidad.

En los sentidos descritos, la indagación en la esfera subjetiva y la construcción de un modelo tentativo respecto del tema, puede ser de gran utilidad para avanzar en una mejor comprensión de la forma en que las políticas y las estrategias de evaluación y apoyo a la calidad son recibidas y absorbidas por los establecimientos, y pensar en mejores formas de promoverlas e implementarlas.

\section{Referencias}

Bailey, B. (2000). The impact of mandated change on teachers. In A. Hargreaves $\& \mathrm{~N}$. Bascia (Eds.), The sharp edge of change: Teaching, leading and the realities of reform (pp. 112-128). London: Falmer Press. 
134 ¿QUÉ ES "EDUCACIÓN DE CALIDAD" PARA DIRECTORES Y DOCENTES? - P. Torche, J. Martínez, J. Madrid y J. Araya

Calderhead, J. (2003). Planning and thinking in junior high school writing lessons: An exploratory study. In M. Kompf y P. M. Denicolo (Dir.), Teacher thinking twenty years on: revisiting persisting problems and advances in education (pp. 53-60). Lisse (Pays-Bas): Swets and Zeitlinger.

Carrasco, A. (2014). Selección de estudiantes y desigualdad educacional en Chile: ¿qué tan coactiva es la regulación que la prohibe? Manuscrito inédito, generado como parte del Proyecto Fonide $\mathrm{N}^{\circ}: 711286$.

Datnow, A. \& Castellano, M. (2000). Teachers responses to success for all: How beliefs, experiences, and adaptations shape implementation. American Educational Research Journal, 37(3), 775-779.

Datnow, A., Hubbard, L., \& Mehan, H. (1998). Educational reform implementation. A co-constructed process [Technical report]. Santa Cruz, CA: Center for Research on Education, Diversity, and Excellence.

Fullan, M. (1991). The new meaning of educational change. New York: Teachers College Press.

Fullan, M. (1993). Change forces: Probing the depths of educational reform. London: Falmer Press.

Glaser, A., Corbin, J., \& Strauss, A. (1990). Basic of grounded theory methods. Beverly Hills, CA.: Sage.

Griffiths, T., Gore, J., \& Ladwig, J. (november 2006). Teachers' fundamental beliefs, commitment to reform, and the quality of pedagogy. Paper preparado para la presentación en el Australian Association for Research in Education Annual Conference Adelaide, Australia

Insunza, J. (2014). Estandarización en educación: anatomía de una deformación. Revista Docencia, 52. Recuperado en: http://www. revistadocencia.cl/visor.php?numeroRevista $=52$

Lara-Cinisomo, S., Fuligni, A. S., Daugherty, L., Howes, C., \& Karoly, L. (2009). A qualitative study of early childhood Educators' beliefs about key preschool classroom experiences. RAND Working Paper No WR- 656. http://dx.doi.org/10.2139/ssrn.1333307

Ley 20.529. Ministerio de Educación. Sistema Nacional de Aseguramiento de la Calidad de la educación parvularia, básica y media y su fiscalización. Publicada en el Diario Oficial el 27 de agosto de 2011.

Raczynski, D. (2011). El paso de la enseñanza básica a la media en estratos bajos: un reto a la igualdad de oportunidades educativas. Proyecto Fonide $\mathrm{N}^{\circ}$ : F511066.

Sarason, S. (1996). The culture of the school and the problem of change. New York: Teachers College Press. 
Sikes, P. J. (1992). Imposed change and the experienced teacher. In M. Fullan \& A. Hargreaves (Eds.), Teacher development and educational change (pp. 36-55). London: Falmer Press.

Universidad Diego Portales. (2014). La voz de los profesores. Recuperado de http://www.educacion2.udp.cl/2014/03/31/escuchar-la-voz-de-losdirectores/

Yero, J. L. (2002). Teaching in mind: How teacher thinking shapes education. Hamilton, MT: MindFlight Publishing.

Recibido: 13/08/2015

Aceptado: 16/10/2015 